\title{
What the Null Energy Condition (and When It May Be Violated) Tells Us about Gravitational Wave Frequencies in/for Relic Cosmology?
}

\author{
Andrew Beckwith \\ Department of Physics, Chongqing University, Chongqing, China \\ E-mail: abeckwith@uh.edu
}

Received April 13, 2011; revised June 12, 2011; accepted June 28, 2011

\begin{abstract}
We introduce a criterion as to the range of HFGW generated by early universe conditions. The 1 to 10 Giga Hertz range is constructed initially starting with what Grupen writes as far as what to expect of GW frequencies which can be detected assuming a sensitivity of $7 \times h \sim 10^{-27}$. From there we examine the implications of an earlier Hubble parameter at the start of inflation, and a phase transition treatment of pre to post Planckian inflation physics via use of inflatons. We close with an analysis of how gravitational constant $\mathrm{G}$ may vary with time, the tie in with the NEC condition and how to select a range of relic GW frequencies. The gravitational frequencies in turn may enable resolving a mis match between the datum that the entropy of the center of the galaxy black hole is greater than the entropy of the present four dimensional universe as we can infer and measure.
\end{abstract}

Keywords: Null Energy Condition, Violation of Null Energy Condition, Cyclic Conformal Cosmology, Entropy, Multiverse

\section{Introduction}

We begin looking at what to expect via the ratio of the energy of relic gravitational waves, over a fixed energy density as a way to quantify the allowed frequency range, and sensitivity allowed, i.e. $1 \mathrm{GHz}$. This permits, if we do it right at looking at a phenomenological treatment of acquisition of the data needed to understand the Hubble parameter, via experimental temperature inputs. Next, if that same Hubble parameter is proportional to the square root of the inflaton potential, the regime of potential change from say $\varphi^{\alpha}$ to $\varphi^{-\alpha}$ as given by Beckwith's [1] adaptation of Weinberg's [2] discussion of scale factor and potentials may signify a phase transition. This outlined set of new results assumes that the inflaton $\varphi$ keeps growing. The choice of $\varphi^{-\alpha}$ is tantamount to the de facto decrease in the scalar field contribution to the scalar potential. It is Beckwith's contention that rising and lowering temperatures as presented in $[1,3]$ are important in determining a range of frequencies from 1 $\mathrm{GHz}$ to $10 \mathrm{GHz}$. The final tabulation of the frequency range is due to looking at uncertainty relations as far as energy and time versus Planck's constant. To get the frequency range tabulated, this inquiry examines the concept of the null energy condition [4]. Fidelity to the null energy condition as assume is combined with $\mathrm{G} \sim \mathrm{G}(\mathrm{t})$. i.e. Is there a gravitiational "constant" parameter having a slight time variation, and a cosmological vacuum energy parameter changing with background temperature as part of what helps give a range of values as to the relic GW frequency? That is the important question asked. Part of this document will be a way to test for inputs into the spectral index, $n_{S}$. That objective is sought, by use of an article by Finelli, Cerioni, and Gruppuso, [5,6]. A case by case analysis of what can be ascertained via such inputs will be presented, with recommendations as to how to get these inputs set up experimentally. The spectral inputs will also be a way to answer a question about comparing entropy as of the universe, and the center of major black holes in the center of galaxies. A mis match which needs resolution.

\section{Vacuum Energy, Sources and Commentary}

We begin first looking at different value of the cosmo- 
logical vacuum energy parameters, in four and five dimensions [7]. i.e. by looking at a five dimensional vacuum energy parameter written as in Equation (1) below.

$$
\left|\Lambda_{5-\mathrm{dim}}\right| \approx C_{1} \cdot\left(1 / T^{\alpha}\right)
$$

This Equation (1) is in contrast with the more traditional four-dimensional version of vacuum energy, minus the minus sign of the brane world theory version. The five-dimensional version is connected with Brane theory and higher dimensions, whereas the four-dimensional version is linked to more traditional De Sitter space-time geometry, as given by Park [8]. Beckwith gives additional refinements [7] as presented in Equation (2)

$$
\Lambda_{4-\mathrm{dim}} \approx c_{2} \cdot T^{\beta}
$$

Right after gravitons are released, one sees a drop-off of temperature contributions to the cosmological constant . Then one writes, for small time values $t \approx \delta^{1} \cdot t_{P}$, $0<\delta^{1} \leq 1$ and for temperatures sharply lower than $T \approx 10^{12}$ Kelvin, a drop off to the present low value of the cosmological constant, Beckwith, writes the inter relationship between the two version of the cosmological vacuum energy, if $n$ is an integer as having the given order of magnitude inter relationsip as given in Equation (3) below [7]

$$
\frac{\Lambda_{4-\operatorname{dim}}}{\left|\Lambda_{5-\operatorname{dim}}\right|}-1 \approx \frac{1}{n}
$$

If there is order of magnitude equivalence between such representations, there is a quantum regime of gravity that is consistent with fluctuations in energy and growth of entropy. An order-of-magnitude estimate will be used to present what the value of the vacuum energy should be in the neighborhood of Planck time in the advent of nucleation of a new universe. The significance of Equation (3) is that at very high temperatures, it reenforces what Beckwith brought up with Tigran Tchrakian, in Bremen, [9] August $29^{\text {th }}, 2008$. i.e., one would like to have a uniform value of the cosmological constant in the gravitating Yang-Mills fields in quantum gravity in order to keep the gauges associated with instantons from changing. When one has, especially for times $t_{1}, t_{2}<$ Planck time $t_{P}$ and $t_{1} \neq t_{2}$, with temperature vaues given so $T\left(t_{1}\right) \neq T\left(t_{2}\right)$, then $\Lambda_{4}\left(t_{1}\right) \neq \Lambda_{4}\left(t_{2}\right)$. i.e. in the regime of high temperatures, one has $T\left(t_{1}\right) \neq T\left(t_{2}\right)$ for times $t_{1}, t_{2}<$ Planck time $t_{P}$ and $t_{1} \neq t_{2}$. The last set of conditions in the prior sentence is such that gauge invariance necessary for soliton (instanton) stability would be broken [10]. That breaking of instanton stability due to changes of $\Lambda_{4}\left(t_{1}\right) \neq \Lambda_{4}\left(t_{2}\right)$ will be where we move from an embedding of quantum mechanics in an analog reality, to the quantum regime. Let us now look at different characterizations of the discontinuity, which is the boundary between analog reality, and Octonian gravity $[10,11]$. Table 1 below is also using material from Barvinsky [12], and will be referred to later.

For times $t>t_{P} \rightarrow$ today, a stable instanton is assumed, along the lines brought up by t'Hooft [13], due to an asymptotic approach to a final, stable $\Lambda_{4-\text { dim }} \approx$ constant value, as the temperature of the universe reaches a net value of $T \approx 3.2 \mathrm{~K}$. That constant for a four dimensional vacuum energy is a very small value, roughly at the value of the cosmological constant given given today. The results given in Table 1 assume a radical drop-off of the cosmological constant after the electroweak transition. That drop off is in line with Kolb's assertion of the net degrees of freedom in space-time drop from about 100 to at most 1000 down to a low value of two, especially if $t>t_{P} \rightarrow$ today in the present era. The supposition is that the value of $\mathrm{N}$ is proportional to a numerical graviton density referred to as $<\mathrm{n}\rangle$, provided that there is a bias toward HFGW, which would mandate a very small value for $V \approx R_{H}^{3} \approx \lambda^{3}$. Furthermore, structure formation arguments, as given by Perkins [14] give evidence that if we use an energy scale, $m$, over a Planck mass value $M_{\text {Planck }}$, as well as contributions from field amplitude $\varphi$, and using the contribution of scale factor behavior $\frac{\dot{a}}{a} \equiv H \approx-m \cdot \frac{\varphi}{3 \cdot \dot{\varphi}}$, where we assume $\ddot{\varphi} \cong 0$ due to inflation

$$
\frac{\Delta \rho}{\rho} \sim H \Delta t \sim \frac{H^{2}}{\dot{\varphi}} \sim\left(\frac{m}{M_{\text {Planck }}}\right) \times\left(\frac{\varphi}{M_{\text {Planck }}}\right) \sim 10^{-5}
$$

At the very onset of inflation, $\varphi \ll M_{\text {Planck }}$, and if $m$ (assuming $\eta=c=1$ ) is due to inputs from a prior universe, we have a wide range of parameter space as to ascertain where $\Delta S \approx \Delta N_{\text {gravitons }} \neq 10^{88}$ [12] comes from. In the next section, we will discuss if it is feasible and reasonable to have data compression of prior universe "information". If $S_{\text {initial }} \sim 10^{5}$ is transferred from a prior uni-

Table 1

\begin{tabular}{cccc}
\hline Time $0 \leq t<<t_{p}$ & Time $0 \leq t<<t_{p}$ & Time $t \geq t_{p}$ & Time $t>t_{p} \rightarrow$ today \\
\hline$\left|\Lambda_{5}\right|$ undefined, $T \approx \varepsilon^{+} \rightarrow T \approx 10^{32} K$ & $\left|\Lambda_{5}\right| \approx \varepsilon^{+}$, & $\left|\Lambda_{5}\right| \approx \Lambda_{4-\text { dim }}$, & $\left|\Lambda_{5}\right| \approx$ huge, \\
$\Lambda_{4-\text { dim }} \approx$ almost $\infty$ & $\Lambda_{4-\text { dim }} \approx$ extremely large & $T$ much smaller than $T \approx 10^{12} K$ & $\Lambda_{4-\text { dim }} \approx$ constant, $T \approx 3.2 K$ \\
\hline
\end{tabular}


verse to our own universe at the onset of inflation, at times less than Planck time $t_{P} \sim 10^{-44}$ seconds, that enough information MAY exit for the preservation of the prior universe's cosmological constants, i.e. $\eta, G, \alpha$ (fine structure constant) and the like. We do not have a reference for this and this supposition is being presented for the first time. Times after $t=10^{-44}$ are not less important. Issues raised in [11-17] are important as to the research protocols

\section{Consider Now What Could Happen with a Phenomenological Model Bases upon the Following Inflection Point i.e. Split Regime of Different Potential Behavior}

$$
V(\varphi)=g \cdot \varphi^{\alpha}
$$

Given the above potential, as in Equation (5), two regimes of space time behavior are examined. Manipulating formalism as given to use by Weinberg [2] we have [1],

$$
V(\phi) \propto \varphi^{|\alpha|} \text { For } t<t_{\text {PLanck }}
$$

Also, we would have

$$
V(\phi) \propto 1 / \varphi^{|\alpha|} \text { For } t \gg t_{\text {PLanck }}
$$

Equations (12) and (13) are predicated on the idea that $\phi$ increases, with $\mathrm{V}$ becoming smaller as Equation (7) approaches the present era. i.e. the potential system vanishes at or before one billion years ago.

The switch between Equations (6) and (7) is not justified analytically. Beckwith [1] designated this divide in behavior as represented by Equations (6) and (7) as the boundary of a causal discontinuity. According to Weinberg [2], if $\in=\frac{\lambda^{2}}{16 \pi G}, H=1 / \in t$ so that one has a scale factor behaving as [2]

$$
a(t) \propto t^{1 / \epsilon}
$$

Then, if [14]

$$
|V(\varphi)| \ll(4 \pi G)^{-2}
$$

there are no quantum gravity effects worth speaking of. i.e., if one uses an exponential potential a scalar field could take the value of, when there is a drop in a field from $\varphi_{1}$ to $\varphi_{2}$ for flat space geometry and times $t_{1}$ to $t_{2}$ [2]

$$
\varphi(t)=\frac{1}{\lambda} \ln \left[\frac{8 \pi G g \in^{2} t^{2}}{3}\right]
$$

Then the scale factors, from Planckian time vary as given in reference [2] as written in Equation (11) below.

$$
\frac{a\left(t_{2}\right)}{a\left(t_{1}\right)}=\left(\frac{t_{2}}{t_{1}}\right)^{1 / \epsilon}=\exp \left[\frac{\left(\varphi_{2}-\varphi_{1}\right) \lambda}{2 \in}\right]
$$

The more $\frac{a\left(t_{2}\right)}{a\left(t_{1}\right)} \gg 1$, then the less likely Equation (11) represents space time conditions requiring quantum gravity. Note those that the way this inflaton as given for a typical Equation (5) behavior

$V(\varphi)=g \cdot \phi^{\alpha} \underset{\text { Time»Planck-Time }}{\longrightarrow} 0^{+}$potential is defined is for a flat, Roberson-Walker geometry, and that if $t_{1}<t_{\text {Planck }}$ then Equation (11) no longer applies. If Equation (11) no longer holds, then a physics observer would observationally finds that one is no longer having any connection with even an Octonionic Gravity regime. The details as to what may be expected via Octonionic gravity and its violation are given in Beckwith [1] as an adaptation of the argument given above. And linked to the next section which is that there is a way to link the phase shift involved in Equations (5)-(11) with a degrees of freedom mapping as given in the next section.

\section{Increase in Degrees of Freedom in the Sub Planckian Regime}

Starting with $[18,19]$

$$
E_{\text {thermal }} \approx \frac{1}{2} K_{B} T_{\text {temperature }} \propto\left[\Omega_{0} \breve{T}\right] \sim \tilde{\beta}
$$

The assumption is that there would be an initial fixed entropy arising, with $\bar{N}$ as a nucleated structure arising in a short time interval as a temperature $T_{\text {temperature }} \varepsilon\left(0^{+}\right.$, $10^{19} \mathrm{GeV}$ ) arrives. One then obtains, dimensionally speaking [18,19]

$$
\frac{\Delta \tilde{\beta}}{\text { dist }} \cong\left(5 k_{B} \Delta T_{\text {temp }} / 2\right) \cdot \frac{\bar{N}}{d i s t} \sim q E_{\text {net-electric-field }} \sim[T \Delta S / \text { dist }]
$$

The parameter, as given by $\Delta \tilde{\beta}$ will be one of the parameters used to define chaotic Gaussian mappings. Candidates as to the inflation potential would be in powers of the inflation, i.e. in terms of $\phi^{N}$, with $N=4$ effectively ruled out, and perhaps $N=2$ an admissible candidate (chaotic inflation). For $N=2$, one gets [11,18], Note that any such entropy as introduced into our universe would have to be consistent with a change given by (if $V^{\prime}=\mathrm{d} V / \mathrm{d} \phi$, where $V$ is an inflaton potential, and dist $=$ distance of Planck length, or more) then Beckwith (2010) (see Equation (14)).

which in the limit of typical chaotic inflation reduces to a more manageable behavior as (see Equation (15)).

Note also in the limit of decline of inflation, Equations 
(14) and (15) imply that eventually one can work with

$$
\lambda \approx 1 / \text { frequency }
$$

If one makes the identification of later time physics, not necessarily in the initial space time regime one no longer has a vacuum energy and/or an inflaton contribution potential at all to contend with, namely

$$
[T \Delta S / \text { dist }] \underset{\text { Large-Time }}{\longrightarrow}[\hbar / \text { dist }] \cdot\left[2 k^{2}\right]^{1 / 2} \approx n
$$

Furthermore, the entropy count is related to what Seth Lloyd (2002) gave in the number of operations as

$$
I=S_{\text {total }} / k_{B} \ln 2=[\text { \#operations }]^{3 / 4} \sim 10^{7}
$$

as implying at least one operation per unit graviton, with gravitons being one unit of information per produced graviton. Note, Smoot (2007) gave initial values of the operations as

$$
\text { [\#operations }]_{\text {initially }} \sim 10^{10}
$$

What would be interesting to investigate would be a tie in to the number of operations, i.e. maybe 10 to the tenth power, and then the evolution of degrees of freedom which will be mentioned below. If the inputs into the inflation, as given by $\phi^{2}$ becomes a random influx of thermal energy from temperature, we will see the particle count on the right hand side of Equations (14) and (15) above a partly random creation of $n_{\text {Particle-Count }}$ which we claim has its counterpart in the following treatment of an increase in degrees of freedom. The way to introduce the expansion of the degrees of freedom from nearly zero, at the maximum point of contraction to having $\mathrm{N}(\mathrm{T}) \sim 10^{2}$ to $10^{3}$ is to define the classical and quantum regimes of gravity in such a way as to minimize the point of the bifurcation diagram affected by quantum processes. [18]. The diagram, in a bifurcation sense would be an application of the Gauss mapping of $[11,18]$.

$$
x_{i+1}=\exp \left[-\tilde{\alpha} \cdot x_{i}^{2}\right]+\tilde{\beta}
$$

In dynamical systems type terminology, one would achieve a diagram, with tree structure looking like what was given by Binous [19], using material written up by Lynch [20] .Now that we have a model as to what could be a change in space time geometry, let us consider what may happen during the Higgs mechanism break down, as given by Beckwith [1] and in very early universe geometry.

\section{The Role Critical Density Plays in Analyzing the Frequency Produced in Relic GW Production}

We are now going to bring up what Grupen [21] brings up about the role of energy density, GW, and also of $\Omega_{G W}$ in terms of setting up frequency changes due to phase shifts in early universe cosmology. To do this, note first that

$$
\rho_{G W}=\left[\frac{h^{2} \omega_{G W}^{2}}{32 \pi \cdot G}\right]
$$

This expression for gravitational wave (energy based) density leads to

$$
\Omega_{G W}=\left[\frac{h^{2} \omega_{G W}^{2}}{12 \cdot H^{2}}\right]
$$

Frequently, if we assume that $G W$ would be very high, we also wind up having that the Hubble parameter $\mathrm{H}$ is also very large. Otherwise, if the $G W$ frequency is low, then Equation (22) is often immeasurably small, a datum which shows up in models of GW generation, in the early universe. As given in [21] having $h \sim 10^{-27} \Leftrightarrow$ $\omega_{G W} \sim 1000 \cdot \mathrm{Hz}$. We can now seriously consider candidates as to the Hubble frequency, as far as phenomenology and to use that to be part of an estimate as far as a permitted range of $G W$ frequencies generated by relic early universe phase transitions. The current idea by [22] is that the Electro weak regime, as designated by Duerrer et al. [22] is by far a greater contributor to $G W$ production, and it is now time to revisit that assumption in detail.

As stated by Sarkar [23], page 481 of his reference, a good temperature based phenomenological treatment of a Hubble parameter would look like

$$
H \equiv 1.66 \sqrt{g^{*}(T)} \cdot\left[T^{2} / M_{P L}\right]
$$

As stated by Beckwith [1] and re duplicated in Equation (20), the argument given is that there would be, if certain conditions are met, a starting low temperature, rapidly rising,with at about the Planck regime of space time a top

$$
\begin{gathered}
{[T \Delta S / \text { dist }]=[\hbar / \text { dist }] \cdot\left[2 k^{2}-\frac{1}{\eta^{2}}\left[M_{\text {Planck }}^{2} \cdot\left[\frac{6}{16 \pi}-\frac{3}{4 \pi}\right] \cdot\left[\frac{V^{\prime}}{V}\right]^{2}-\frac{3}{4 \pi} \cdot M_{\text {Planck }}^{2} \cdot\left[\frac{V^{\prime \prime}}{V}\right]\right]\right]^{1 / 2}} \\
{[\Delta S]=[\hbar / T] \cdot\left[2 k^{2}-\frac{1}{\eta^{2}}\left[M_{\text {planck }}^{2} \cdot\left[\left[\frac{6}{4 \pi}-\frac{12}{4 \pi}\right] \cdot\left[\frac{1}{\phi}\right]^{2}-\frac{6}{4 \pi} \cdot\left[\frac{1}{\phi^{2}}\right]\right]\right]\right]^{1 / 2} \sim n_{\text {partick-Count }}}
\end{gathered}
$$


degrees of freedom expression of about $\left.g^{*}(T)\right|_{\text {Maximum }} \sim$
1000 , for the temperature reaching $T \mid T_{P L}$ in 1000 , for the temperature reaching $\left.T\right|_{\text {Maximum }} \sim T_{P L}$ in value from an initially much lower value. This is also a
datum, which if we reach $g^{*}(T) \quad \sim 1000$ would be in sync with Sarkar's [23]

$$
H \sim \sqrt{8 \pi V(\phi) / 3 M_{P L}^{2}}
$$

The matter to consider, would be, frankly, that looking at the following expression of energy flux being re formulated for each universe. I.e. start with the Alcubierre's [24] formalism about energy flux, assuming that there is a solid angle for energy distribution $\Omega$ for the energy flux to travel through. [24,25] looking at a change of energy if

$$
\frac{\mathrm{d} E}{\mathrm{~d} t}=[\lim r \rightarrow \infty]\left[\frac{r^{2}}{16 \pi}\right] \oint\left|\int_{-\infty}^{t} \Psi_{4} \mathrm{~d} t^{\prime}\right|^{2} \cdot \mathrm{d} \Omega
$$

The expression $\Psi_{4}$ is a Weyl scalar which we will write in the form of [24,25]

$$
\begin{aligned}
\Psi_{4}= & -\frac{1}{4} \cdot\left[\partial_{t}^{2} h^{+}-2 \partial_{t} \partial_{r} h^{+}+\partial_{r}^{2} h^{+}\right] \\
& +\frac{i}{4} \cdot\left[\partial_{t}^{2} h^{x}-2 \partial_{t} \partial_{r} h^{x}+\partial_{r}^{2} h^{x}\right]
\end{aligned}
$$

Our assumptions are simple, that if the energy flux expression is to be evaluated properly, before the electro weak phase transition, that time dependence of both $h^{+}$ and $h^{x}$ is miniscule and that initially $h^{+} \approx h^{x}$, so as to initiate a rewrite of Equation (21) above as [24]

$$
\Psi_{4} \cong-\frac{1}{4} \cdot\left[+\partial_{r}^{2} h^{+}\right] \cdot(-1+i)
$$

The upshot, is that the initial energy flux about the inflationary regime would lead to looking at [25]

$$
\left|\int_{-\infty}^{t} \Psi_{4} \mathrm{~d} t^{\prime}\right| \approx\left|\frac{1}{2} \cdot\left[+\partial_{r}^{2} h^{+}\right]\right| \cdot\left(\tilde{n} \cdot t_{\text {Planck }}\right)
$$

This will lead to an initial energy flux at the onset of inflation which will be presented as [25]

$$
\frac{\mathrm{d} E}{\mathrm{~d} t}=\left[\frac{r^{2}}{64 \pi}\right] \cdot\left|+\partial_{r}^{2} h^{+}\right|^{2} \cdot\left[\tilde{n} \cdot t_{\text {Planck }}\right]^{2} \cdot \Omega
$$

If we are talking about an initial energy flux, we then can approximate the above as [25]

$$
E_{\text {initial-flux }} \cong\left[\frac{r^{2}}{64 \pi}\right] \cdot\left|+\partial_{r}^{2} h^{+}\right|^{2} \cdot\left[\tilde{n} \cdot t_{\text {Planck }}\right]^{3} \cdot \Omega_{\text {effective }}
$$

Inputs into both the expression $\left|\partial_{r}^{2} h^{+}\right|$, as well as $\Omega_{\text {effective }}$ will comprise the rest of this document, plus our conclusions. The derived value of $\Omega_{\text {effective }}$ as well as $E_{\text {initial-flux }}$ will be tied into a way to present energy per graviton, as a way of obtaining $n_{f}$. The $n_{f}$ value so obtained, will be used to make a relationship, using $\mathrm{Y}$. J. Ng's entropy [15] counting algorithm of roughly $S_{\text {entropy }} \sim n_{f}$. We assert that in order to obtain $S_{\text {entropy }} \sim n_{f}$ from initial graviton production, as a way to quantify $n_{f}$, that a small mass of the graviton can be assumed. For the sake of convenience, one can write [24-27]

$$
\left|\partial_{r}^{2} h^{+}\right| \sim k^{2} h^{+}
$$

So, then [25]

$$
E_{\text {initial-flux }} \sim\left[\frac{r^{2}}{64 \pi}\right] \cdot k^{4} \cdot\left[h^{+}\right]^{2} \cdot\left[\tilde{n} \cdot t_{\text {Planck }}\right]^{3} \cdot \Omega_{\text {effective }}
$$

For our purposes, we shall call $r \sim l_{\text {Planck }} \propto 10^{-34} \mathrm{~cm}$, $t_{\text {Planck }} \sim 10^{-44} \mathrm{sec}, \Omega_{\text {effective }}$ an effective cross sectional area as to the emission of gravitons, and $k$ defined as a physical wave vector. L. Crowell stated that $G W$ would undergo massive red shifting, [28,29] Needless to state, the value of $k$ to consider would be for the $\mathrm{GHz}$ band of $G W[26,27]$.

$$
\left(k \approx k_{G W}\right)^{2} \gg\left|\frac{1}{a} \cdot \frac{\mathrm{d}^{2} a}{\mathrm{~d} \eta^{2}}\right|
$$

Also, for the frequencies of $[27,30] 10^{9}-10^{10} \mathrm{~Hz}$, then

$$
h \sim h_{\text {rms }} \sim 10^{-30}-10^{-34}
$$

Namely, if a net acceleration is such that $a_{\text {accel }}=$ $2 \pi k_{B} c T / \eta$ as mentioned by Verlinde [31,32] as an Unruh result, and that the number of "bits" is

$$
n_{B i t}=\frac{\Delta S}{\Delta x} \cdot \frac{c^{2}}{\pi \cdot k_{B}^{2}} \approx \frac{3 \cdot(1.66)^{2} g^{*}}{\left[\Delta x \cong l_{p}\right]} \cdot \frac{c^{2} \cdot T^{2}}{\pi \cdot k_{B}^{2}}
$$

This Equation (30) has a $\mathrm{T}^{2}$ temperature dependence for information bits, as opposed to [15,23,32,33]

$$
S \sim 3 \cdot\left[1.66 \cdot \sqrt{\tilde{g}_{*}}\right]^{2} T^{3} \sim n_{f}
$$

Should the $\Delta x \cong l_{p}$ order of magnitude minimum grid size hold, then when $\mathrm{T} \sim 10^{19} \mathrm{GeV}[31,32]$

$$
n_{\text {Bit }} \approx \frac{3 \cdot(1.66)^{2} g^{*}}{\left[\Delta x \cong l_{p}\right]} \cdot \frac{c^{2} \cdot T^{2}}{\pi \cdot k_{B}^{2}} \sim 3\left[1.66 \cdot \sqrt{\tilde{g}_{*}}\right]^{2} T^{3}
$$

The situation for which one has $[31,32] \quad \Delta x \cong l^{1 / 3} l_{\text {planck }}^{2 / 3}$ with $\quad l \sim l_{\text {planck }}$ corresponds to $n_{B i t} \propto T^{3}$ whereas $n_{\text {Bit }} \propto T^{2}$ if $\Delta x \cong l^{1 / 3} l_{\text {planck }}^{2 / 3} \gg l_{\text {planck }}$.

Here, we make ths assumption that either $\tilde{n} \sim n_{B i t} \propto$ $T^{2}$ or $\tilde{n} \sim n_{\text {Bit }} \propto T^{3}$ per unit volume of phase space with the temperature $T$ varying from a low value to up to $10^{34}$ Kelvin ( Planck temperature scale).

We will next reference as to conditions permitting re- 
lease of $\tilde{n} \sim n_{\text {Bit }} \propto T^{2}$ or $\tilde{n} \sim n_{\text {Bit }} \propto T^{3}$ per unit volume of phase space, while also noting a way to also identify dimensional contributions to relic particle conditions. Taking into account, as given by U. Sarkar [23] for relic Graviton production, as a function of extra dimensions we can denote by

$$
n_{\text {relic-graviton-production }}(T) \sim T^{2} \cdot[T / M]^{d+2}
$$

We can though, if we wish to reconcile Equation (38) with release of $\tilde{n} \sim n_{\text {Bit }} \propto T^{2}$ or $\tilde{n} \sim n_{\text {Bit }} \propto T^{3}$ look at temperature dependence of the scaled mass value, $\mathrm{M}$, Furthermore, if a phase transition exists, as mentioned by Subir Sarkar the following change is revealing recall Subir Sarkar's [34] 2001 investigation of a simple choice of variant of the standard chaotic inflationary potential given by

$$
V \equiv V_{0}-c_{3} \phi^{3}+\frac{1}{2} \lambda \cdot \phi^{2} \cdot \rho^{2}+\cdots
$$

Sarkar treated the inflaton as having a varying effective mass, with an initial value of effective mass of $m_{\phi}^{2}=\frac{\mathrm{d}^{2} V}{\mathrm{~d} \phi^{2}}$ given a before and after phase transition value of [34]

$$
\begin{aligned}
m_{\phi}^{2}= & -\left.6 c_{3} \cdot\langle\phi\rangle\right|_{\text {Before-phase-transition }} \stackrel{\text { phase-transition }}{\longrightarrow} \\
& -6 c_{3} \cdot\langle\phi\rangle+\left.\lambda \cdot \Sigma^{2}\right|_{\text {after-phase-transition }}
\end{aligned}
$$

This is, when Hunt and Sarkar [34] did it, with $\lambda=\kappa \cdot \mathrm{m}^{2} / M_{P}^{2}$ as a coupling term. This would also affect the spectral index value, and it also would be a way to consider an increase in inflation based entropy The value of $\mathrm{M}$ so given in Equation (38) we believe is connected with an appropriate choice of the details of the phase transition alluded to in Equation (40) above.

There are two ways to reconcile information from Equation (40) as far as a temperature dependence affecting $M$, as I see it, and connecting it with Equation (40) and-release of $\tilde{n} \sim n_{B i t} \propto T^{2}$ or $\tilde{n} \sim n_{\text {Bit }} \propto T^{3}$ First [23]

$$
T^{2} \cdot[T / M]^{d+2} \sim T^{2} \Leftrightarrow M \propto T
$$

This will as we will present below apparently implying $\Delta x \cong l^{1 / 3} l_{\text {planck }}^{2 / 3} \gg l_{\text {planck }}$ It so happens that Equation (41) with a direct temperature dependence of a net mass $\mathrm{M}$ is equivalent to the production of gravitons/relic particles as dictated by an initially fixed starting temperature, i.e. making the $\Delta x \cong l^{1 / 3} l_{\text {planck }}^{2 / 3}$ with $l \sim l_{\text {planck }}$ corresponds to $n_{B i t} \propto T^{3}$ whereas $n_{B i t} \propto T^{2}$ if $\Delta x \cong l^{1 / 3}$ $l_{\text {planck }}^{2 / 3} \gg l_{\text {planck }}$. The minimum grid size being possibly of the form $\Delta x \cong l^{1 / 3} l_{\text {planck }}^{2 / 3} \gg l_{\text {planck }}$ implies a fixed set of initial space parameters, with temperature not affected by extra dimensions. Secondly, one can make the following approximation as obeying [23]

$$
T^{2} \cdot[T / M]^{d+2} \sim T^{3} \Leftrightarrow M \propto T^{\alpha},|\alpha|<1
$$

This may correspond to implying changing the minimum length fluctuation to. $\Delta x \cong l^{1 / 3} l_{\text {planck }}^{2 / 3}$ with $l \sim$ $l_{\text {planck }}$

\section{Summary as to What Is Known, and Not Known about the Null Energy Condition in Cosmology, and Information Exchange between Prior to Present Universes}

As stated in [4], the NEC is linked to the following, i.e. look at the general null energy condition first. The null energy condition stipulates that for every future-pointing null vector field (for all of the GR) $\vec{k}$

$$
\rho=T_{a b} k^{a} k^{b} \geq 0
$$

With respect to a frame aligned with the motion of the matter particles, the components of the matter tensor take the diagonal form, in Euclidian space that

$$
\mathrm{T}^{\mathrm{ab}}=\left[\begin{array}{cccc}
\rho & 0 & 0 & 0 \\
0 & P & 0 & 0 \\
0 & 0 & P & 0 \\
0 & 0 & 0 & P
\end{array}\right]
$$

The simplest statement of the Null energy condition is that he null energy condition stipulates that density and momentum obey

$$
\rho+P \geq 0
$$

i.e. the equation of state to consider is, if $w \leq-1$, then if what [1] suggests is true, then there will be a reason to consider the relative import of Equations (43)-(45) in terms of contributions. i.e. we do have problems with the idea of variance of the cosmological constant, $G$. We also will build upon the consequences of $w \leq-1$, We can generalize this idea to initial domain wall physics. Spherical geometry does not violate the NEC. Further domain wall physics may lead to a break down of the NEC [4]. We also refer to a treatment of the NEC if we look at an effective Friedman equation as given by [2], as seen by

$$
\begin{aligned}
H_{a}^{2}= & {\left[\frac{\dot{a}}{a}\right]^{2}=\frac{8 \pi G}{3} G \cdot \rho+\left(\frac{\kappa^{2}}{24 \cdot(3+n)}\right) . } \\
& {[2+n \cdot[1-3 \cdot(v-w)]] \cdot \rho^{2}-e }
\end{aligned}
$$

The scaling done in this situation has [2], especially if $\mathrm{e}$ is a constant in Equation (46) leading to using,

$$
\rho=a^{-3 \cdot[1+w]}
$$

As stated in [16]. We expect that there will be flat 
space geometry almost in the beginning of the early big bang. I.e. this will lead to Equation (47), if $w<-1$ implying that $\rho=a^{-3 \cdot[1+w]} \sim a^{+\varepsilon} \rightarrow 0^{+}$if there is a violation of the NEC. As quoted from [18]. i.e. as seen in a colloquium presentation done by Dr. Smoot in Paris [35] (2007); he alluded to information theory as to how much is transferred between a prior to the present universe in terms of information "bits",

1) Physically observable bits of information possibly in present Universe - $10^{180}$.

2) Holographic principle allowed states in the evolution/development of the Universe - $10^{120}$.

3) Initially available states given to us to work with at the onset of the inflationary era - $10^{10}$.

4) Observable bits of information present due to quantum/statistical fluctuations - $10^{8}$.

Our guess is as follows. That the thermal flux accounts for perhaps $10^{10}$ bits of information. These could be transferred from a prior universe to our present, and that there could be $10^{120}$ minus $10^{10}$ bytes of information suppressed during the initial bozonification phase of matter right at the onset of the big bang itself. Beckwith [37] stated criteria as far as graviton production, and a toy model of the universe. If one has Equation (42) shut off due to $w<-1$, so then that $\rho=a^{-3 \cdot[1+w]} \sim a^{+\varepsilon} \rightarrow 0^{+}$ occurs, then the causal discontinuity so references in [18] by Beckwith et al, will have major consequences as far as a away to determine if gravitons have a small mass, and if there is a way to determine if a prior universe has contribution as to the information transferred as to the present universe. We will now assume, that the catastrophe given as $\rho=a^{-3 \cdot[1+w]} \sim a^{+\varepsilon} \rightarrow 0^{+}{ }_{\mathrm{n}}$

\section{Determination If the NEC Is Valid Is Essential as Establishing a Necessary Condition for Transfer of Information from a Prior Universe to Today's Cosmos}

How to do this? i.e. how to determine if, as an example there is a thermal, flux from a prior universe carrying prior universe information? We will briefly revisit a first principle introduction as to inflaton fluctuations in the beginning which may be part of how to obtain experimental falsifiable criterion. From Weinberg [2], we can write, from page 192 - 193, if an inflaton potential $V(\phi) \sim M^{4+\alpha} \phi^{-\alpha}$ then, the inflaton potential has the fluctuation behavior given by [1]

$$
\delta \phi \sim t^{\gamma}
$$

Then, this result for Equation (48) assumes

$$
\gamma=-.25 \pm \sqrt{\frac{1}{16}-\frac{(6+\alpha) \cdot(1+\alpha)}{(2+\alpha)^{2}}}
$$

The resulting contributions to the $\mathrm{CMBR}$, if worked out, and also connections to gravitational wave astronomy can be used to pin point an eventual CMBR physics behavior as referred to by Beckwith [1] and its relationship to falsifiable experimental tests of the NEC.

\section{How to Calculate the Spectral Index $n_{S}$ for a Dissipative Regime of the Inflaton?}

We are largely borrowing in this introduction from work done by Finelli, Cerion, and Gruppuso [2,3] and we will introduce the motivation behind their work as well as the actual Spectral index $n_{S}$. To begin with look at what Finelli et al $[5,6]$ postulate as to the case of warm inflation. i.e. as given by $[1,5,6]$, if the equation of state $\omega_{F}=p_{F} / \rho_{F}$ is linked to $\left[3 H \rho_{F} \cdot\left(1+\omega_{F}\right)\right] \cong \Gamma \dot{\phi}^{2}$ so then we get the statement of

$$
\ddot{\phi}+[3 H+\Gamma] \dot{\phi}+\left(\frac{\partial V}{\partial \varphi}\right)=0
$$

We can count the term given as $[3 H+\Gamma] \dot{\phi}$ as a damping term, as well as consider the slow roll value of

$$
[3 H+\Gamma] \dot{\phi}+\left(\frac{\partial V}{\partial \varphi}\right) \cong 0
$$

The above dynamics, if $V_{\phi \phi}=\frac{\mathrm{d}^{2} V}{\mathrm{~d} \phi^{2}}$, and $\Gamma=\Gamma_{0}$.

$$
\begin{aligned}
& {\left[\frac{\phi}{\phi_{0}}\right]^{b} \cdot\left[\frac{\sqrt[4]{\rho_{F}}}{M}\right]^{C}, \text { and }} \\
& \qquad \gamma=\frac{\Gamma}{3 H}, \varepsilon=-\frac{\dot{H}}{H^{2}}, \quad \eta_{\phi \phi}=\frac{V_{\phi \phi}}{3 H^{2}}
\end{aligned}
$$

For the sake of convenience, we can use $V_{\phi \phi} \sim$ constant, i.e. the quadratic scalar potential. But this is a special case of what we will refer to later. If so, then the equations for perturbations, inflaton perturbations, $Q_{\phi}$, $Q_{F}$ as respectively the inflaton, and the fluid fluctuations leads to initial conditions of

$$
\begin{aligned}
& Q_{\phi \mid k} \cong \exp \left[-i k \cdot\left(\tau-\tau_{i}\right)\right] /\left(a^{1+\frac{3}{2} \gamma} \sqrt{2 k}\right) \\
& Q_{F \mid k} \cong \exp \left[-i k \cdot\left(\tau-\tau_{i}\right)\right] /\left(a^{1+\frac{3}{2}\left(1+\omega_{F}\right) \cdot(1-g)} \sqrt[4]{4 k^{2} \omega_{F}}\right)
\end{aligned}
$$

The upshot is that one gets the following as far as a running index $[1,5,6]$

$$
n_{S}-1 \cong-3 \gamma^{*}+\frac{2}{1+\gamma^{*}} \eta^{*}-6 \cdot\left[\frac{1+\frac{4}{3} \gamma^{*}+\frac{1}{2}\left[\gamma^{*}\right]^{2}}{1+\gamma^{*}}\right] \cdot \varepsilon^{*}
$$


Here, the * factor is for values of the parameters when the cosmological evolution crosses a radius defined by $\left(\mathrm{k}_{-}=\mathrm{a}_{-} \mathrm{H}_{-}\right)$. In [2] there are two tables as far as inputs/ out puts into running index, which have to take into account several constraints. i.e. when one has, as was stated a situation for which [1]

$$
\Gamma=\Gamma_{0} \cdot\left[\frac{\phi}{\phi_{0}}\right]^{b} \cdot\left[\frac{\sqrt[4]{\rho_{F}}}{M}\right]^{C}=\text { const }^{*}
$$

Either $b=C=0$, which is possible, or one could have, if $b \neq 0, C \neq 0$, a situation for which one can have

$$
\left[\frac{\phi}{\phi_{0}}\right]^{b} \cdot\left[\frac{\sqrt[4]{\rho_{F}}}{M}\right]^{C}=\text { const }
$$

What if one had, $\phi_{0}$ being a present day, very small value of a scalar field [1]

$$
\phi=\text { const } \cdot \phi_{0} \cdot\left[\frac{M}{\sqrt[4]{\rho_{F}}}\right]^{C / b}
$$

We can probably assume in all of this that $\mathrm{M}$ as a mass scale is fixed. When the author looks at Equation (57), it appears to be implying the relative value of density, i.e. $\rho_{F}$ varies with time. i.e. if one looked at the Octonian gravity formation regime we could look at variation of looking maybe like $\rho_{F} \sim H_{\text {observed }}^{2} / G$. The term about the relationship of [36], where a is a constant, and $g^{*}(T)$ is the number of degrees of freedom,

$$
\rho_{F} \sim H_{\text {observed }}^{2} / G \approx 4 \pi \cdot a T^{4} g^{*}(T) / 3 c^{2}
$$

There are two different scenarios as far as temperature build up and how it affects $g^{*}(T)$, and also initial temperatures.

\section{1. ${ }^{\text {st }}$, Version of Classical/Standard Cosmology Treatment of the Start of Inflation. i.e. the Ultra High Temperature Regime to Cooler Temperatures}

Here, as given by Kolb and Turner [37], $g^{*}(T)$ has a peak of about 100 - 120 during the electro weak regime, and that there is allegedly little sense in terms of modeling of talking about $g^{*}(T)$ before the electro weak regime. What it means? In so many words, we would then have $\rho_{F}$ undefined before the electro weak regime. $\varphi$ would then be undefined before the electro weak regime. It does mean that at the start of the electro weak regime, we would see an increasing $\varphi$. Which is the opposite of what we see. i.e. we need $\varphi$ decreasing. Meaning that either $g^{*}(T)$ is defined before the electro weak phase transition, or Equation (58) no longer holds.

How to tie in the entropy with the growth of the scale function? Racetrack models of inflation, assuming far more detail than what is given in this simplistic treatment provide a power spectrum for the scalar field given by $[38,39]$

$$
P \sim \frac{1}{150 \pi^{2}} \cdot \frac{V(\varphi)}{\epsilon}
$$

This is very close to what Giovannini puts in, [40], and $n_{S}$ being the spectral index

$$
P(k)=\frac{8}{3 M_{P}^{4}} \cdot\left(\frac{V(\varphi)}{\epsilon}\right)_{k=a H} \approx k^{n_{S}-1}
$$

This Equation (60) result is assuming a slow roll parameter treatment with $\in \ll 1$, and for $t>t_{p}$. An increase in scalar power, is then proportional to an increase in entropy via use of the following equation.

$$
\left|\frac{\Delta E}{l_{P}^{3}}\right| \sim\left|\frac{\Delta P \in 150 \pi^{2}}{l_{P}^{3}}\right| \approx|\Delta S|
$$

This Equation (61) result presumes that there exists awell defined $V(\varphi)$ before the start of the Planck time interval. That is, if we want to make the equivalent statement $|\Delta S| \sim \Delta \mathrm{n}$ for [15] a numerical relic count, as done by $\mathrm{Ng}$ [12] does not tell us where the relic particles came from, As we also note in [20] we can employ Sherrer $k$ essence arguments [3] as to how to form relic particles without using a potential explicitly for times less than Planck time interval.

\section{2. $1^{\text {st }}$, New Treatment of the Start of Inflation, i.e. First Low Temperature, Then Ultra High Temperature Regime to Cooler Temperatures (Low to High Then Low Temperature Evolution)}

This model of low temperature to higher temperatures involves using the initial analysis, except that one has $\mathrm{g}^{*}(\mathrm{~T})$ defined initially as of about 2 in pre Planckian space time, rising to about 100 to a peak possible value of 1000, as of Planck time, [18] and then from there declining. The initial temperature would be low, which would rise to a peak temperature, i.e. Planck temperature value, and then subsequently moving to values seen today. This scenario is outlined in [1], and has the advantage of explaining at least before to about the Planck time interval, how Equation (54) could resort to a rising temperature. Now, having said, that, what is the advantage toward having Equation (55) set as a constant with a rising inflaton value and with $b \neq 0, C \neq 0$ ?

\section{Comparing the Reacceleration of the Universe, via Deceleration Parameter, Initially and Finally Speaking}

The use of Equation (62) below to have re-acceleration in 
this formulation is dependent upon "heavy gravity" as the rest mass of gravitons in four dimensions has a small mass term. This equation below is developed by Beckwith [4042]

$$
q=-\frac{\ddot{a} a}{\dot{a}^{2}}
$$

We wish next to consider what happens not a billion years ago, but at the onset of creation itself. If a correct understanding of initial graviton conditions is presented, it may add more credence to the idea of a small graviton mass, in a rest frame, Here, we are making use of refining the following estimates. In what follows, we will have even stricter bounds upon the energy value (as well as the mass) of the graviton based upon the geometry of the quantum bounce, with a radii of the quantum bounce on the order of $l_{\text {Planck }} \sim 10^{-35}$ meters $[43,44]$. So then the mass of a graviton implies a wavelength to the graviton as can be written as given in Equation (63) below.

$$
\begin{aligned}
& \left.m_{\text {graviton }}\right|_{\text {RELATIVISTIC }}<4.4 \times 10^{-22} h^{-1} \mathrm{eV} / c^{2} \\
& \Leftrightarrow \lambda_{\text {graviton }} \equiv \frac{\eta}{m_{\text {graviton }} \cdot c}<2.8 \times 10^{-8} \text { meters }
\end{aligned}
$$

For looking at the onset of creation, with a LQG bounce; if we look at $\rho_{\max } \propto 2.07 \cdot \rho_{\text {planck }}$ for the LQG quantum bounce with a value put in for when $\rho_{\text {planck }} \approx 5.1 \times 10^{99}$ grams $/$ meter $^{3}$, where the effective energy is

$$
E_{\text {eff }} \propto 2.07 \cdot l_{\text {Planck }}^{3} \cdot \rho_{\text {planck }} \sim 5 \times 10^{24} \mathrm{GeV}
$$

Then, taking note of this, one is obtaining having scaled entropy of $S \equiv E / T \sim 10^{5}$ when one has an initial Planck temperature $T \approx T_{\text {Planck }} \sim 10^{19} \mathrm{GeV}$. One then needs to consider, if the energy per given graviton is, if a frequency $v \propto 10^{10} \mathrm{~Hz}$ and $E_{\text {graviton-effective }} \propto 2 \cdot h v \approx 5 \times$ $10^{-5} \mathrm{eV}$, then there is a minimum entropy value we can write as.

$$
\begin{aligned}
& S \equiv E_{\text {eff }} / T \sim \\
& {\left[10^{38} \times E_{\text {graviton-effective }}\left(v \approx 10^{10} \mathrm{~Hz}\right)\right] /\left[T \sim 10^{19} \mathrm{GeV}\right] \approx 10^{5}}
\end{aligned}
$$

Having said that, the $E_{\text {graviton-effective }} \propto 2 \cdot h v \approx 5 \times 10^{-5}$ $\mathrm{eV}$ is $10^{22}$ greater than the rest mass energy of a graviton if $E \sim m_{\text {graviton }}[$ red - shift $\sim .55] \sim\left(10^{-27} \mathrm{eV}\right)$ grams is taken.

\section{Now, for Permitted Frequency Ranges for the Relic Graviton}

As given by Hambler [45] for the effective Friedman equation, on his books pages 318 - 319. In the procedure which will be written up, we can set $\xi \approx a_{0}$ with $a_{0}$ as defined by what is known as the running gravitational coupling in the vicinity of the ultra violet fixed point as given in equation 9.1 of Hambler [45].

$$
G(t)=G \cdot\left[1+C_{\xi} \cdot\left(\frac{t}{\xi}\right)^{v}\right]
$$

The time varying value of $G$ does lead to an effective density as given by

$$
\rho_{\text {effective }}(t)=\frac{G(t)}{G} \cdot \rho(t)
$$

If one is making an analysis of the effective energy, as given by an analysis in part given by $\mathrm{Ng}$ [15] and Beckwith [46]

$$
\begin{aligned}
& \left|\frac{\Delta E}{l_{P}^{3}}\right| \sim|\Delta S| \approx \Delta n_{\text {relic-gravition }} \propto \eta \Delta \omega_{\text {relic-graviton }} \\
& \approx \eta \cdot\left[1+C_{\xi} \cdot\left(\frac{t}{\xi}\right)^{v}\right] \cdot \omega_{\text {relic-graviton }}
\end{aligned}
$$

The relic graviton frequency so described would be from $\left[E_{\text {graviton-effective }} \propto 2 \cdot h v \approx 5 \times 10^{-5} \mathrm{eV}\right]$ which is $10^{22}$ greater than the rest mass energy of a graviton, taking $\omega_{\text {relic-graviton }} \sim E_{\text {graviton-effective }} / \eta$, with $\left[E_{\text {graviton-effective }}\right.$ $\left.\propto 2 \cdot h v \approx 5 \times 10^{-5} \mathrm{eV}\right]$ is over $10^{22}$ times greater than the rest mass energy of a graviton. The spread in the frequencies would be given by the factor $1+C_{\xi} \cdot\left(\frac{t}{\xi}\right)^{v}$. Let us for the sake of completeness analyze where this came from. The Friedman equation, as given by Hambler [46] with $\mathrm{k}$ the curvature factor, and

$$
\frac{k}{a^{2}(t)}+\left[\frac{\dot{a}(t)}{a(t)}\right]^{2}=\frac{8 \pi G}{3} \cdot\left[1+C_{\xi} \cdot\left(\frac{t}{\xi}\right)^{v}\right] \cdot \rho(t)+\frac{1}{3 \xi^{2}}
$$

In short, we get, a variance in the Friedman equation. The variance in the Friedman equation appears to be linked to a density variance as given below.

$$
\Delta \rho_{F} \sim 4 \pi \cdot a(\Delta T)^{4} g^{*}(\Delta T) / 3 c^{2}
$$

As mentioned earlier, we have, in Equation (68) and also in Equation (69) a duration of time for which there is a build up of temperature, of the magnitude $\Delta T$ just before the inflationary era, and that the time factor is tied into $1+C_{\xi} \cdot\left(\frac{t}{\xi}\right)^{v}$ of Equation (68) It means that in the context of relic graviton production, that the frequency range as of $G W$ production is, indeed nearly a delta func- 
tion. Why is this delta function behavior significant? If one looks at a frequency for relic GW in terms of an upper bound as to $G W$ frequency, i.e. if frequency $f>f_{*}=4.4 \times 10^{-9} \mathrm{~Hz}$ as given by Buoanno [47], then the bound to Equation (71) follows. i.e.

$$
h_{0}^{2} \Omega_{G W} \leq 4.8 \times 10^{-9} \cdot\left(f / f_{*}\right)^{2}
$$

One gets a bias toward low frequencies, and this is accentuated by an estimate which needs to be looked at and questioned, namely, if there is, according to Buonanno, [47] purely adiabatic evolution of the universe, Here, we have that $a_{0}$ is todays value of the cosmological constant, whereas $a_{*}, f_{*}$ are initial scale factor and frequency values for the production of GWs as given by Buonnanno [47]

$$
f=f_{*} \cdot\left(a_{*} / a_{0}\right)
$$

If the bias toward low frequency values is removed and we look at generation of say having $a_{*} \sim 10^{-25} a_{0}$ for nearly relic conditions, one gets astonishingly high initial values for $f_{*}$, i.e.

$$
f_{*}=f \cdot\left(a_{0} / a_{*}\right) \sim 10^{25} \cdot f_{\text {Today }} \propto 10^{35} \mathrm{~Hz}
$$

Note that next to Equation (73) we calculated de facto $10^{22}$ times greater than the rest mass energy of a graviton for relativistic graviton energy. i.e. what was being predicted by the adiabatic approximation has a value of, already about $10^{25}$ times larger for the frequency.

Of course, though, an adiabatic approximation is nonsense for the initial phases of the universe, but it is still indicative as to what could be the starting point to a legitimate inquiry

We should note that researchers as of China and the United States have project work on answering the feasibility of this sort of measurement. [48] Should there be a way to make such a measurement, some of the issues so referred to, i.e. the feasibility of semi adiabiatic approximations can be considered. Secondly, and most importantly, if the genesis of initial $G W$ production is within the Planck regime as so mentioned above, for the initial $10^{35} \mathrm{~Hz}$ value for frequency will be congruent with extremely tiny starting geometries.

\section{1. $1^{\text {st }}$ Part of Conclusions, What to Make of Pre-Planckian Physics, in Terms of What to Measure via a GW Detector}

We will initially quote part of the conclusion as of [1] here, and add more to it.

Finelli et al [6] claims that $\gamma^{*} \geq 0.01$ does not match observations, with $\gamma=\Gamma / 3 H$. We gave arguments in the prior session as to the feasibility of having $\Gamma$ as a constant, which often appears to create serious difficul- ties. If one has $\Gamma$ as a constant, with rising inflaton value, $\phi$ up to Planck time interval we have a natural reason for $\Lambda_{4-\operatorname{dim}}$ varying, and also $\rho_{F} \neq$ const, assuming that with rising inflaton value, $\phi$ up to Planck time interval?

$1^{\text {st }}$ we have a natural reason for $\Lambda_{4 \text {-dim }}$ varying, and also $\rho_{F} \neq$ const varies with $g^{*}(T)$ varying from 2 to 1000 before the electro ${ }_{2}$ weak era, and $\rho_{F} \neq$ const having $S \sim 3 \cdot\left[1.66 \cdot \sqrt{\tilde{g}_{*}}\right]^{2} T^{3}$ increasing in a net temperature increase up to at least $10^{5}$ from nearly zero, initially.

Having said that, we should also revisit what was brought up in [18] namely in how likely we are to be able to get such measurements. Doing so, asks the question of if gravitons have a small rest mass, and that leads to the second real issue to consider. From [18] we wrote for how to isolate the effects of a 4 dimensional graviton with rest mass. If one looks at if a four dimensional graviton with a very small rest mass included [18] we can write how a graviton would interact with a magnetic field within a $G W$ detector.

$$
\frac{1}{\sqrt{-g}} \cdot \frac{\partial}{\partial x^{v}} \cdot\left(\sqrt{-g} \cdot g^{\mu \alpha} g^{v \beta} F_{\alpha \beta}\right)=\mu_{0} J^{\mu}+J_{\text {effective }}
$$

where for $\varepsilon^{+} \neq 0$ but very small

$$
F_{[\mu v, \alpha]} \sim \varepsilon^{+}
$$

The claim which A. Beckwith made [18] is that

$$
J_{\text {effective }} \cong n_{\text {count }} \cdot m_{4-D \text {-Gravtion }}
$$

As stated by Beckwith, in [18], $m_{4-D-\text { Gravtion }} \sim 10^{-65}$ grams, while $n_{\text {count }}$ is the number of gravitons which may be in the detector sample. What would be needed to do would be to try to isolate out an appropriate stress energy tensor contribution due to the interaction of gravitons with a static magenetic field $T^{u v}$ assuming a non zero graviton rest mass.

The details of the $n_{\text {count }}$ would be affected by the degree of the graviton mass, the frequency range and a whole lot of other parameters, but the key point would be in finding a specified frequency range, which the author claims for relic gravitons is almost a spike, as well as their energy level. From there, using some of the details brought up in this document would be relevant, in a program of action as to how to get necessary experimental confirmation. We hope to do so, as soon as circumstances permit. We also seek to find ways to confirm what t'Hooft brought up in [13].

\section{2. $2^{\text {nd }}$ Part of Conclusions, the Future Game Plan}

\section{1. $2^{\text {nd }}$ Part of Conclusions: Information Theory Considerations, and Solving the}




\section{Problem of a Black Hole in the Center of the Galaxy Having More Entropy than the Entire Universe}

$1^{\text {st }}$ : If Entropy has for a single black hole, say $10^{100}$ versus a value of $10^{88}$ for the entire universe. An example of such is given in a NASA news service [49] and is noteworthy, since we will claim that the black holes in such galaxies do have more than four dimensions. This is crucial. Note that these numbers are given by Carroll, as in reference [50]. The problem though is that no amount of conformal rescaling of space time geometry [51] will itself help us reconcile has for a single black hole, say $10^{100}$ versus a value of $10^{88}$ for the entire universe. It is useful though tor review the suggestion of what is implied by conformal cyclic cosmology.

The heart of the hypothesis is in what Penrose called conformal re scaling, namely looking at what Paul Tod wrote for a spatial metric, to re scale almost infinite geometry back to a new big bang [51]

$$
\breve{g}_{a b}=\breve{\Omega}^{2} g_{a b}
$$

In so many words, after a near infinite expansion of the universe, re scale the "infinite expansion" via a conformal re scaling back to a new big bang. Also, Tod [51] writes

$$
\breve{\Omega} \cong \exp H t
$$

The interesting addition to this hypothesis is given by Tod, [52] and Penrose to read as having a positive parameter

$$
\Lambda=3 \mathrm{H}^{2}
$$

As stated by Tod [51], Penrose writes, namely that Quote:

In (Penrose, [52] 2008), Penrose presents a picture of the very remote future with positive-as a physical worldin which proper-time plays no role. He remarks that all stars will have completed their evolution and either collapsed to form black holes or been swallowed up by the massive black holes at the centres of galaxies. Black holes themselves will eventually decay by the Hawking process and the content of the universe will very largely be just electromagnetic and gravitational radiation, both of them massless felds. To complete the picture of a world from which proper-time has vanished, Penrose hypothesises that all massive particles eventually either decay to radiation or lose their mass in some unspecified way.

Still though there is no way that conformal mappings or conformal re scaling can make the following mapping, using what was presented by Lloyd, [33] in terms of information theory

$$
\begin{aligned}
& 10^{100} \underset{\text { conformal-mapping- } \cos \text { mo } \log y}{\longrightarrow} \text { Value } \\
& \equiv I=S_{\text {total }} / k_{B} \ln 2=[\text { \# operations }]^{3 / 4} \sim 10^{7}
\end{aligned}
$$

This is to be compared to Entropy of black holes in the center of galaxies, e.g. our own, can be greater than the entropy associated with the entire four dimensional observational universe, as given by [50] writes that the entropy of the central black hole of the galaxy is

$$
\begin{aligned}
& S_{\text {Black-hole-center-of-galaxy }} \sim 10^{90} \cdot\left[\frac{M}{10^{6} M_{\text {solar-mass-of-sun }}}\right]^{2} \\
& >\text { entropy-of -observed-universe }
\end{aligned}
$$

Equation (81) is for a single black hole at the center of the galaxy. If there are over $10^{6}$ galaxies, the question is what happens to $\sim 10^{99}$ units of entropy per Galaxy? Of a single black hole as opposed to $10^{88}$ to $10^{90}$ for the general universe.

$2^{\text {nd. }}$ The difference between $10^{106}$ units of entropy, versus $10^{88}$ for the entire universe (Carroll, 2004) [50] can only be resolved if Black holes are 5 dimensional (or higher dimensional objects).

\section{2. $2^{\text {nd }}$ Part of Conclusions: Arrow of Time/and 5 Dimensional Black Holes}

What Beckwith became convinced of, due to these arguments is that Black holes, and other information collection portals have to be considered in higher dimensions. To do this, look at Re define a general entropy which may exist in five dimensions, so that if the starting point is to look at (Penrose, 2011) [52] in terms of a temperature value, the vacuum energy, and also entropy, directly. From the book (Penrose, 2011) [52], if $G=1$ and $\Lambda$ is a vacuum energy and from Penrose, 2011 [52]

$$
\begin{aligned}
S_{\text {entropy-black-hole }} & \equiv \frac{\tilde{A}}{4} \propto S_{\text {general-entropy-4dim }}=\frac{\tilde{A}_{\Lambda}}{4} \\
& \equiv \frac{12 \pi}{4 \Lambda}=\frac{3 \pi}{\Lambda} \Leftrightarrow T_{\Lambda}=\frac{1}{2 \pi} \sqrt{\frac{\Delta}{3}}
\end{aligned}
$$

Then, using Table 1, we can have, the two different limiting values for entropy in four and five dimensions. The five dimensional entropy would initially be enormous, whereas there is a different interpretation for the magnitude of four dimensional entropy.

$$
\begin{aligned}
& S_{\text {general-entropy-5dim }}=\frac{\tilde{A}_{\Lambda}}{4} \equiv \frac{12 \pi}{4\left|\Lambda_{5 \text {-dim }}\right|} \approx \infty \\
& \Leftrightarrow S_{\text {general-entropy-4dim }}=\frac{\tilde{A}_{\Lambda}}{4} \equiv \frac{12 \pi}{4\left|\Lambda_{4-\mathrm{dim}}\right|} \\
& \approx \text { tiny-value }
\end{aligned}
$$


So, how does one justify this result? Doing it implies coming up with a multi verse for containment of the four dimensional universe. i.e. demoting the present universe we are in as one out of perhaps billions of (Tegmark 2003) [53] level four universes contributing to entropy. In a manner which is still being worked out, Beckwith is attempting to take the Penrose suggestion of conformal recycling, but to do it in a way which avoids the problem as implied by Equations (80) and (81). Note that the five dimensional representation of black holes would be similar to what is presented in reference [54]. Equation (83) would probably point to a large degree of entropy dumped from four dimensional universes by black holes, extending from each 4 dimensional universe into a fifth dimension.

\section{3. $3^{\text {rd }}$ Part of Conclusions, What to Look for in Term of Observations and Information Transfer?}

Confirming or denying the importance of the multiverse would be crucial. The idea that black holes may have a 5 dimensional embedding space, as also part of their representation also means taking into consideration the following as given by Penrose (2011) [52], i.e. Penrose claims that right at the time of the CMBR, that the entropy per baryon is $10^{9}$ to $10^{10}$. Similarly, Penrose claims that the entropy per baryon is about $10^{21}$ today. What the entropy per 'particle' before the turn on of CMBR, closer to the big bang would be is not stated by Penrose, but it probably would be far lower than $10^{9}$.

Secondly, Penrose's cyclic conformal cosmology is for times up to about $10^{-31}$ seconds is allegedly implying (Penrose, 2011) in the last stated reference for [52] that the product of a [distance measure] times a [momentum measure] is an invariant quality. A reduction/ increase in information present in a distance measure $\Leftrightarrow$ increase/reduction in information present in a distance measure i.e. the key point being Equation (80) and then Equation (81) would still have to be explained. Even if the entropy per "particle" or clumping of "information" were dramatically lower than $10^{9}$, what information may be transferred from prior universe embedding of our present universe, should be reconciled with 1 to $10 \mathrm{GHz}$ relic $G W$ being generated initially.

The information content implied by Equation (63) to Equation (65), in terms of multiverses would need to be verified experimentally. Beckwith's guess is that violation of the Null energy condition will be important as well as a slowly time varying $G(t)$ value. This behavior would start off by an initial energy step being proportional to the inverse of a varying initial time step as given in Equation (84) below.

$$
\Delta E \approx 1 / \Delta t \sim \Delta E_{\text {thermal }} \approx \frac{1}{2} k_{B} T_{\text {temperature }} \propto \Delta T
$$

Beckwith submits that the smallness of the initial time step $\Delta t$, as given of the order of Planck Time, reflecting the variance in temperature $\Delta t$ is a consequence of the Null Energy condition. In turn, Equation (84) suggests a necessary re do of the Penrose cyclic conformal cosmology suggestion [54], which will eventually lead to an indirect proof of Tegmarks [53] multiverse (level four) hypothesis. The transition given by Equation (84), i.e. a phase transition, from a pre quantum regime, perhaps represented by a multi verse [53] to a regime of space time given by Octonionic geometry, as given by Appendix A below. This transition, and the information transfer as alluded to in the document would be where a violation of the Null Energy condition would be of paramount importance. The violation of the null energy condition would be in the transfer from Pre Octonionic to Octonionic geometry, with Octonionic geometry, signifying the initial regime of quantum gravity as given in appendix A below [56].

\section{Acknowledgements}

This work is supported in part by National Nature Science Foundation of China grant No. 11075224 The author wishes to thank Dr. Fangyu Li for his repeated hospitality in Chongquing, PRC, as well as Stuart Allen, of international media associates whom freed the author to think about physics, and get back to his work.

\section{References}

[1] A. W. Beckwith, "What Violations of the Null Energy Condition Tell Us about Information Exchange between Prior to Present Universes? How to Obtain Spectral Index Confirmation?" http://vixra.org/abs/1102.0030

[2] S. Weinberg, "Cosmology," Oxford University Press, New York, 2008

[3] A. W. Beckwith, "Is Nature Fundamentally Continuous or Discrete, and How Can These Two Different but Very Useful Conceptions Be Fully Reconciled? (Condensed Version)," 2011. http://vixra.org/abs/1102.0019

[4] P. J. Steinhardt and D. Wesley, "Dark Energy, Inflation and Extra Dimensions," Physical Review D, Vol. 79, No. 10, 2009, pp. 1010-1021. doi:10.1103/PhysRevD.79.104026

[5] F. Finelli, A. Cerioni and A. Gruppuso, "Is a Dissipative Regime For the Inflation in Agreement with Observations?" In: J. Dumarchez, Y. Giraud-Heraud and J. T. T. Van, Eds., Cosmology, Guoi Publishers, Vietnam, 2008, pp. 283-286.

[6] F. Finelli, A. Cerioni and A. Gruppuso, "Is a Dissipative Regime during Inflation in Agreement with Observa- 
tions?" Physical Review D, Vol. 78, No. 2, 2008, Article ID: 021301.

[7] A. W. Beckwith, "Relic High Frequency Gravitational waves from the Big Bang, and How to Detect Them," American Institute of Physics Conference Proceedings, Vol. 1103, 2009, pp. 571-581.

[8] D. K. Park, H. Kim and S. Tamarayan, "Nonvanishing Cosmological Constant of Flat Universe in Brane World Scenarios," Physics Letters B, Vol. 535, No. 1-2, 2002, pp. 5-10. doi:10.1016/S0370-2693(02)01729-X

[9] T. Tchrakian and D. H. Presentation, "Gravitating Yang-Mills Fields," Models of Gravity in Higher Dimensions, Bremen, 25-29 August 2008.

[10] A. W. Beckwith, "Implications for the Cosmological Landscape: Can Thermal Inputs from a Prior Universe Account for Relic Graviton Production?" American Institute of Physics Conference Proceedings, Vol. 969, 2008, pp. 1091-1102.

[11] A. W. Beckwith, "How to Use the Cosmological Schwinger Principle for Energy Flux, Entropy, and 'Atoms of Space Time', for Creating a Thermodynamics Treatment of Space-Time," Journal of Physics: Conference Serie, Vol. 306, 2011, Article ID: 012064.

[12] A. Barvinsky, A. Kamenschick and A. Yu, "Thermodynamics from Nothing: Limiting the Cosmological Constant Landscape," Physical Review D, Vol. 74, 2006, Article ID: 121502 .

[13] G. T. Hooft, "How Instantons Solve the U(1) Problem," Physical Reports, Vol. 142, No. 6, 1986, pp. 357-387. doi:10.1016/0370-1573(86)90117-1

[14] D. Perkins, "Particle Astrophysics," Oxford Master series in Particle Physics, Astrophysics, and Cosmology, Oxford, 2005

[15] Y. J. Ng, “Article: Spacetime Foam: From Entropy and Holography to Infinite Statistics and Nonlocality," Entropy, Vol. 10, No. 4, 2008, pp. 441-461. doi: $10.3390 / \mathrm{e} 10040441$

[16] L. Glinka, "Quantum Information from Graviton-Matter Gas," Sigma, Vol. 3, 2007, p. 13

[17] W. D. Goldberger, "Effective Field Theories and Gravitational Radiation," In: F. Bernardeau, C. Grogean and J. Dalibard, Eds., Session 86, Elsevier, Particle Physics and Cosmology, the Fabric of Space time, Les Houches, Oxford, 2007, pp. 351-396.

[18] A. W. Beckwith, F. Y. Li, et al., "Is Octonian Gravity Relevant near the Planck Scale," Nova Book company, 2011. http://vixra.org/abs/1101.0017

[19] S. Lynch, "Dynamical Systems with Applications Using Mathematica," Birkhauser, Boston, 2007.

[20] H. Binous, "Bifurcation Diagram for the Gauss Map from the Wolfram Demonstrations Project," 2010

[21] C. Grupen, "Astroparticle Physics," Springer-Verlag, Berlin, 2005.

[22] R. Durrer and M. Rinaldi, "Graviton Production in Non-Inflationary Cosmology," Physical Review D, Vol. 79, No. 6, 2009, Article ID: 063507.
doi:10.1103/PhysRevD.79.063507

[23] U. Sarkar, "Particle and Astroparticle Physics, Series in High Energy Physics, Cosmology, and Gravitation," Taylor \& Francis, Boca Racon, 2008

[24] M. Alcubierre, "Introduction to 3+1 Numerical Relativity, International Series of Monographs on Physics," Oxford University Press, Oxford, 2008.

[25] A. W. Beckwith, "Energy Content of Gravition as a Way to Quantify both Entropy and Information Generation in the Early Universe," Journal of Modern Physics, Vol. 2, No. 2, February 2011, pp. 58-61.

[26] F. Li, M. Tang and D. Shi, "Electromagnetic Response of a Gaussian Beam to High Frequency Relic Gravitational Waves in Quintessential Inflationary Models," Physical Review D, Vol. 67, 2003, pp. 1-17. doi:10.1103/PhysRevD.67.104008

[27] F. Li and N. Yang, "Phase and Polarization State of High Frequency Gravitational Waves," Chinese Physics Letters, Vol. 236, No. 5, 2009, Article ID: 050402, pp. 1-4.

[28] L. Crowell, "Quantum Fluctuations of Space-Time," World Scientific Series in Contemporary Chemical Physics, Singapore City, Vol. 25, 2005.

[29] L. Crowell, private communication with the author

[30] F. Y. Li, N. Yang, Z. Y. Fang, R. M. L. Baker Jr., G. V. Stephenson and H. Wen, "Signal Photon Flux and Background Noise in a Coupling Electromagnetic Detecting System for High Frequency Gravitational Waves," 2009. http://vixra.org/abs/0907.0030

[31] A. W. Beckwith and L. Glinka, "The Arrow of Time Problem: Answering if Time Flow Initially Favouritizes One Direction Blatantly," Prespacetime Journal, Vol. 1, No. 9, November 2010, pp. 1358-1375.

[32] E. P. Verlinde, "On the Origins of Gravity and the Laws of Newton," 2010. arXiv:1001.0785v1[hep-th]

[33] L. Seth, "Computational Capacity of the Universe", Physical Review Letters, Vol. 88, No. 23, 2002, Article ID: 237901.

[34] P. Hunt and S. Sakar, "Multiple Inflation and the WMAP 'glitches'," Physical Review D, Vol. 70, No. 10, 2004, Article ID: 103518.

[35] G. Smoot; "CMB Observations and the Standard Model of the Universe 'D. Chalonge' School,' 11th Paris Cosmology Colloquium, Paris, 18 August 2007.

[36] R. H. Sanders, "Observational Support for the Standard Model of the Early Universe," In: E. Papantonopoulos, Ed., Lecture Notes in Physics, Springer-Verlag, Berlin-Heidelberg, Vol. 653, 2005, pp. 105-137.

[37] E. Kolb and S. Turner, "The Early Universe," Westview Press, Chicago, 1994.

[38] E. Komatsu1, J. Dunkley, et al., "Five-Year Wilkinson Microwave Anisotropy Probe Observations: Cosmological Interpretation," The Astrophysical Journal Supplement Series, Vol. 180, No. 2, 2009, p. 330.

[39] M. Giovannini, "A Primer on the Physics of the Cosmic Microwave Background," World Scientific, Pte. Ltd, Singapore City, 2008. 
[40] A. W. Beckwith, "Entropy Growth in the Early Universe, and the Search for Determining if Gravity is Classical or Quantum Foundations (Is Gravity a Classical or Quantum Phenomenon at Its Genesis 13.7 Billion Years Ago?)" Relativity and Cosmology, 2010. http://vixra.org/abs/0910.0057

[41] A. W. Beckwith, "Deceleration Parameter $Q(Z)$ in Four and Five Dimensional Geometries, and Implications of Graviton Mass in Mimicking DE in Both Geometries," Beyond the Standard Model, 2010. http://vixra. org/abs/1002.0056

[42] A. W. Beckwith, "Applications of Euclidian Snyder Geometry to the Foundations of Space-Time Physics," Electronic Journal of Theoretical Physics, Vol. 7, No. 24, 2010, pp. 241-266.

[43] M. Maggiore, "Gravitational Waves," Theory and Experiment, Oxford University Press, Oxford, Vol. 1, 2008.

[44] D. Valev, "Neutrino and Graviton Rest Mass Estimations by a Phenomenological Approach," Aerospace Research in Bulgaria, Vol. 22, 2008, pp. 68-82.

[45] H. Hamber, "Quantum Gravitation, The Feynman Path Integral Approach," Springer-Verlag, Berlin, 2009.

[46] A. W. Beckwith, "Entropy Production and a Toy Model as to Irregularities in the CMBR Spectrum," Pres Space Time Journal, 2011. http://vixra.org/abs/1102.0007

[47] A. Buonanno, "Gravitional Waves," Les Houches, Editors Bernardeau, Grojean, Dalibard, Elsevir, Oxford, 2007, pp. 3-48.

[48] R. C. Woods, R. M. L. Baker Jr., F. Y. Li, G. V. Stephenson, E. W. Davis and A. W. Beckwith, "A New

\section{Appendix A. Primer on Octonionic Mathematics, and Its Significance}

An octonion $\mathrm{x}$ is expressed [Bisht, B. Pandey and O. P. S. Negi, 2009] [56] as a set of eight real numbers

$$
\begin{aligned}
x & =e 0 \times 0+e 1 \times 1+e 2 \times 2+e 3 \times 3+e 4 \times 4 \\
& +e 5 \times 5+e 6 \times 6+e 7 \times 7=e 0 \times 0+\sum_{A=1}^{7} e_{A} x_{A}
\end{aligned}
$$

where $e \mathrm{~A}(\mathrm{~A}=1,2, \cdots, 7)$ are imaginary octonion units and $\mathrm{e} 0$ is the multiplicative unit element. Set of octets $(e 0$, $e 1, e 2, e 3, e 4, e 5, e 6, e 7)$ are known as the octonion basis elements and satisfy the following multiplication rules

$$
\begin{gathered}
e 0=1 ; e 0 e A=e A e 0=e A \\
e A e B=-\_A B e 0+f A B C e C .(A, B, C=1,2, \cdots,
\end{gathered}
$$

Theoretical Technique for the Measurement of HighFrequency Relic Gravitational Waves," 2011. http://vixra.org/abs/1010. 0062

[49] NASA News Service, 2011. http://www.nasa.gov/mission_pages/swift/bursts/monster -black-holes.html

[50] S. Carroll, "Spacetime and Geometry," Addison Wesley, Boston, 2004.

[51] P. Tod, "Spanish Relativity Meeting (ERE 2009) Penrose's Weyl Curvature Hypothesis an Conformally-Cyclic Cosmology," Journal of Physics: Conference Series, Vol. 229, 2010, Article ID: 012013.

[52] R. Penrose, "Before the Big Bang. An Outrageous New Perspective and Its Implications for Particle Physics," Proceedings of EPAC, Edinburgh, 26-30 June 2006, pp. 2759-2763.

[53] M. Tegmark, "Parallel Universes. Not Just a Staple of Science Fiction, Other Universes Are a Direct Implication of Cosmological Observations," Scientific American, Vol. 288, No. 5, 2003, pp. 40-51. doi:10.1038/scientificamerican0503-40

[54] C. Stelea, K. Schleich and D. Witt, "Charged KaluzaKlein Double-Black Holes in Five Dimensions," Physical Review D, Vol. 83, No. 8, 2011, Article ID: 084037. doi:10.1103/PhysRevD.83.084037

[55] A. W. Beckwith, "Octonionic Gravity Formation, Its Connections to Micro Physics," Open Journal of Micro Physics, Vol. 1, No. 1, May 2011, pp. 13-18.

[56] P. S. Bisht, B. Pandey and O. P. S. Negi, Fizika B (Zagreb), Vol. 17, 2008, p. 405

Here, the structure constants $f A B C$ is completely antisymmetric

The multiplication rules in Equation (A3) and its corresponding lead to the generators ei obey the commutation relation;

$$
[e j, e k]=2 f j k l \text { el }
$$

Furthermore, we have that the structure constants $\mathrm{fABC}$ is completely antisymmetric and takes the value 1 for the following combinations,

$$
\begin{aligned}
f A B C=+1 ; \text { if }(A B C)= & (123),(471),(257),(165), \\
& (624),(543),(736) .
\end{aligned}
$$

Equation (A4) above, with a build up in terms of the Octonionic basis referred to in Equations (A1)-(A3), according to Pushpa, P. S. Bisht, T. Li, and O. P. S. 
Negi Leads to a generalization for the Gell Mann Matrices symmetry from $\mathrm{SU}(2)$ to $\mathrm{SU}(3)$ we replace three Pauli spin matrices by eight Gellmann $\lambda_{i}$ matrices. Then Equation (A4) will be built up as

$$
\left[\_\mathrm{j}, \lambda \_k\right]=2 \mathrm{Fjkl} \_\lambda 1(8 j, k, l=1,2,3,4,5,6,7,8)
$$

The generalization as to expanding Equation (1) above, if $\left[e_{A}, e_{B}\right]=\left[\lambda_{A}, \lambda_{B}\right]$

As according to Pushpa, Bisht, Li, and Negi would lead to associator structure

$$
(x, y, z)=(x y) z-x(y z), \text { for any } x, y, z
$$

Implying, depending upon the build up of entries into space and momentum [26]

$$
\left[x_{j}, p_{i}\right]=-\beta \cdot\left(l_{\text {Planck }} / l\right) \cdot \eta T_{i j k} x_{k}
$$

Here, in doing so, the scaling factor, for Planck energy term $E_{\text {Planck }}$

$$
\beta=1+\left[E / E_{\text {Planck }}\right]^{\gamma}
$$

whereas the $T_{i j k}$ is a structure term in some respects acting similar to the basis one used for the Gell Mann matrices, in part dependent upon how the momentum and spatial matrix entries are built up. 